\title{
Seamless Browsing of Visual Contents in Shared Learning Environments
}

\author{
Marco Loregian \\ University of Milano-Bicocca \\ via Bicocca degli Arcimboldi 8, \\ 20126 Milano, Italy \\ loregian@disco.unimib.it
}

\author{
Kresimir Matkovic \\ VRVis Research Center \\ Donau-City-Strasse 1 \\ A-1220 Vienna, Austria \\ matkovic@vrvis.at
}

\author{
Thomas Psik \\ Technical University Vienna \\ Argentinierstrasse 8 \\ A-1040 Vienna, Austria \\ tomp@ims.tuwien.ac.at
}

\begin{abstract}
This paper presents a new interaction technique for browsing large visual information bases in a collaborative environment. The ATELIER project deals with learning environments for architecture and interaction design students. Since students' attitude is to collect large amounts of data, pictures and videos in particular, an important issue is how to keep information organized according to intuitive criteria has been seriously taken into account. This paper shows how we managed to align different approaches for browsing large image sets and describes the interfaces used to seamlessly switch from one view to another. We also implemented search facilities based both on color layout and on keywords taken from an ontology. Integration was achieved by means of physical handles, i.e., barcodes.
\end{abstract}

\section{Introduction}

ATELIER (Architecture and Technologies for Inspirational Learning Environments) is an EU-funded project that is part of the Disappearing Computer initiative ${ }^{1}$. The aim of the project is to build a digitally enhanced environment supporting creative learning processes in architecture and interaction design education. The ATELIER studio is a room in which students feel comfortable with technology and which they can fully configure as to fit their needs [1]. Several technologies were adopted to build a complex system to support students, and make their work easier, so they can focus more on their actual tasks rather than on learning how to use the system. The ATELIER software infrastructure provides means for devices and applications to communicate. It also includes a hyper-media database (HMDB) that is used to store all digital material collected by the students.

The architecture students involved in the project attend the Academy of Fine Arts in Vienna. The teachers at the

\footnotetext{
${ }^{1}$ http://www.disappearing-computer.net
}

academy do not encourage the use of the conventional software (i.e., CAD, modelling, visualization). The focus at the academy is on arts, seeing things differently, experimenting and improving creativity. Students are assigned collaborative projects that require trips to collect material and then they have to develop very unique and original visions. The importance of having some room for sharing their knowledge becomes evident as soon as the final goal of their work - concept elaboration and the realization of some artifacts, i.e., models and presentations - is taken into account.

Students in creative learning environments work daily with thousands of pictures they collected for their projects. It is important that they can easily find and access their data, and also that they have means to sort, annotate and browse their digital material. The contents that they create and collect (e.g., digital pictures, handmade sketches, notes, videos) can be analyzed in different ways and from different points of view since the same item can be given different meanings according to the context in which it is used. These considerations, and the fact that the ATELIER project is part of the Disappearing Computer initiative, led us to develop a number of alternative ways of accessing visual data.

Detailed descriptions of how students work were already given in previous papers $[1,9]$ as well as descriptions of some of the tools we developed $[2,11]$. The main contribution of this paper is the description of how very different search facilities were combined in a seamless way by means of physical handles. The benefit of such an effort is that it improves content management and reduces the need for system training to novice users.

\section{The ATELIER workspace}

Architecture students mainly work on projects devoted to understanding an existent environmental situation and to finding new meanings, ideas and further developments to it. Students' work usually implies outdoor, in field, explorations [9]. Whenever they get back to the ATELIER room, the material gathered is brought into the system, so they can 


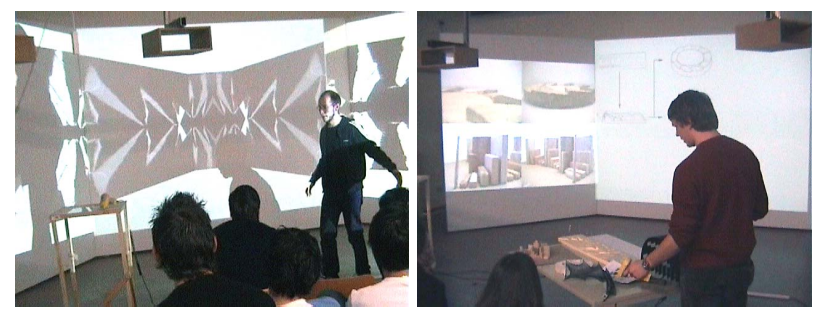

Figure 1. Presentations in the ATELIER room

start to explore the data, to combine new information with the items already present in the system, and to elaborate concepts for their preentations.

The ATELIER room contains several large screens where the projection planes can be adjusted to form a closed space or a flat wide screen or anything between those extremes (see Figure 1). Creating an immersive environment is important both for learning and for presenting. Moreover, each screen may be used to project up to nine different picures or movie clips at the same time. The students may then customize the room for their presentations.

Our aim is to build a system that is easy to access and to manipulate even by non-expert users by suppling interfaces that at the same time improve system usability and hide technical issues from end-users. For this reason we decided to associate physical handles (i.e., barcodes) to the actions repeatedly performed to configure the environment [1] and to digital items stored in the HMDB.

Barcodes are easy to produce and inherit all affordances of paper on which they are printed (i.e., lightness, flexibility, etc.). However, barcodes have some downsides as well, such as their fragility and visual intrusiveness.

The main tool to configure the ATELIER environment is the Configuration Poster. It is a cardboard poster, A3sized, on which actions and the corresponding barcodes are printed. A student can use each of the projection displays that are available in the room to present pictures or movies, by first selecting the barcode for a particular screen and then scanning the barcode for the image or video to be played. The configuration created, meaning the content displayed on each screen, can then be saved. As a consequence, a new barcode is automatically printed on a page with a short description of the configuration (e.g., date and time when it was created). The system will reproduce the same configuration whenever the barcode is read. This functionality enables users to use several displays and other presentational devices and to test new configuration with no fear of losing their work.

As an example of making use of physical handles to manipulate digital items in the environment, we provide a barcode for each uploaded image (like in [12]). After uploading images to the HMDB these so-called Thumbnail Pages

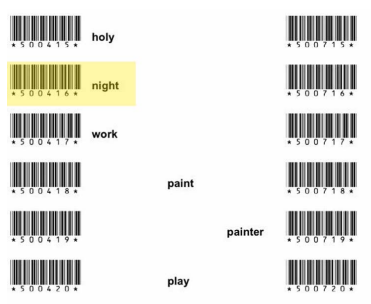

Figure 2. A page of the Ontology Booklet.

can be printed out. For each "upload session" the users get pages with thumbnails of each picture they added, and a barcode printed underneath each of them. The barcodes may then be used as a starting point for all actions in the ATELIER environment.

Once data has been collected and stored in the HMDB it has also to be classified, in order to be useful: a single group of students (3-6 people) can collect a huge amount of pictures and videos. The average size for the HMDB at the end of each project is about $16 \mathrm{~Gb}$ : about 800 media files for a one-day field trip are stored. The reference point for organizing all the material is an ontology.

The ontology can be shortly defined as a hierarchically organized set of keywords that can be applied to give descriptions of items in the HMDB. Different kinds of relation among terms are captured (e.g., the part-of or the $i s-a$ relations). We built several facilities around the ontology [2] and the way it is dynamically built and maintained is described in [9]. The ontology is of paramount importance for learning processes but, in order to be useful, it has also to be easily accessible. We initially created a PC GUI tool to access the ontology and provide meta-information for the HMDB items, we added more "tangible" solutions later.

The ontology is printed on a booklet (see Figure 2) to have a reference ready-at-hand whenever it is needed. Barcodes are printed next to each keyword so that it can be selected. For example, a keyword can be toggled on or off from the description of an image by simply reading its proper barcode. This mechanism provides a way to manage meta-information in a tangible way. The downside is that if new keywords are added to the ontology or the ontology structure is modified then a new barcode set - even if compatible and overlapping with the older one - must be printed.

To actually use and present images or clips that are in the HMDB each user must also be able to retrieve a particular image. We implemented several methods to access the HMDB and perform various "search-and-browse" interactions to assist the students in their work. 


\section{Seamless search applications}

We distinguish two ways of accessing visual data. Sometimes users look for a particular image, and sometimes they just want to browse through the data to get some inspiration. Architecture students, like all art students, often use image browsing as an inspirational method [2]. Browsing can be especially inspiring if images are grouped in a way that the grouping criterion is not visible at first sight [7]. Searching for similarities in images that are totally different at first sight can trigger new ideas, and help students in seeing things differently.

Apart from the already mentioned Thumbnail Pages we developed additional methods to access contents in the HMDB; the Tangible Image Query and the Ontology Search. The Tangible Image Query [11] is an application that looks for images with a similar color layout and it is more suitable for inspirational browsing described above, while the Ontology Search represents a keyword-based search method enhanced by the use of physical handles.

Each method has peculiar qualities, while the combination and simultaneous use of both creates a powerful access mechanism for visual data.

The most common way to search for some information in a large database is through a keyword-based search engine. In our case we wanted to get rid of typing, therefore we studied other means to support interactive searches. Keywords are printed, in a hierarchical tree structure, on a paper booklet. Each keyword has two barcodes associated (see Figure 2), one printed on the left-hand side that is used to query the database, and one printed on its right-hand side that is used to toggle the keyword on or off. The Ontology Search supports different ways to search for images in the HMDB. Since the ontology encompasses various relations among concepts, a number of terms can be grouped; for example: searching for animal might also return pictures having horse, cow or chicken attached to them. Another search possibility is to directly use a picture as an input for the search, so that pictures having a similar description - i.e., pictures described using an overlapping set of keywords will be returned.

The Image Query implements a query by example approach, by providing a search criterion in terms of similarity to another picture instead of in terms of a formal description of a picture (i.e. referring to its name, content, author or other meta-information). In this case the first problem is to find a suitable definition for similar. Different criteria can be adopted, for our prototype we relied on the Visual Image Query by Matkovic et al. [10] based on the color-layout similarity. This sometimes yields surprising and unexpected results: images that have little in common at first sight are displayed next to each other. Such combinations encourage lateral thinking by students and the start of new creative

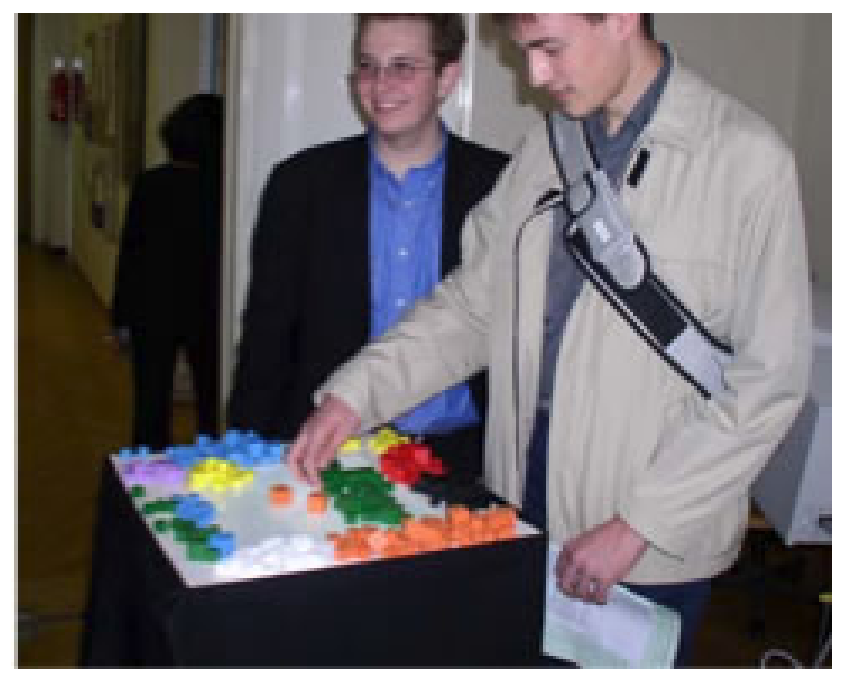

Figure 3. The Tangible Image Query table.

processes. We also created a tangible user interface called the Tangible Image Query [11] (Figure 3). Interactions take place on a table that has a semi-transparent white glass top surface with a set of colored objects (e.g., wooden cubes) with which a color layout query can be created (detailed description in [11]).

\section{Integration scenario}

Although both of the above described methods work well independently, their seamless integration provides a more powerful visual-data access mechanism. Since both tools are integrated in the software infrastructure for the ATELIER environment, and use the same HMDB, we had only to develop an interaction mechanism to make them work together. We designed a special set of configuration sheets - the Query Control Sheets (see Figure 4) — which are used to control both applications. The users interact with the system using the Tangible Image Query table, the Ontology Booklet and the Control Sheet, which are both attached to the table. We will now describe with a practical example how all the features fit together.

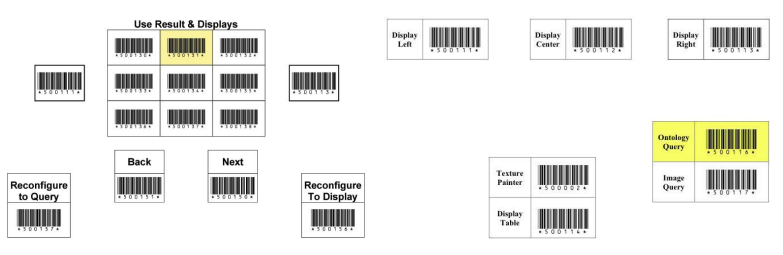

(a)

(b)

Figure 4. The Query Control Sheets. 


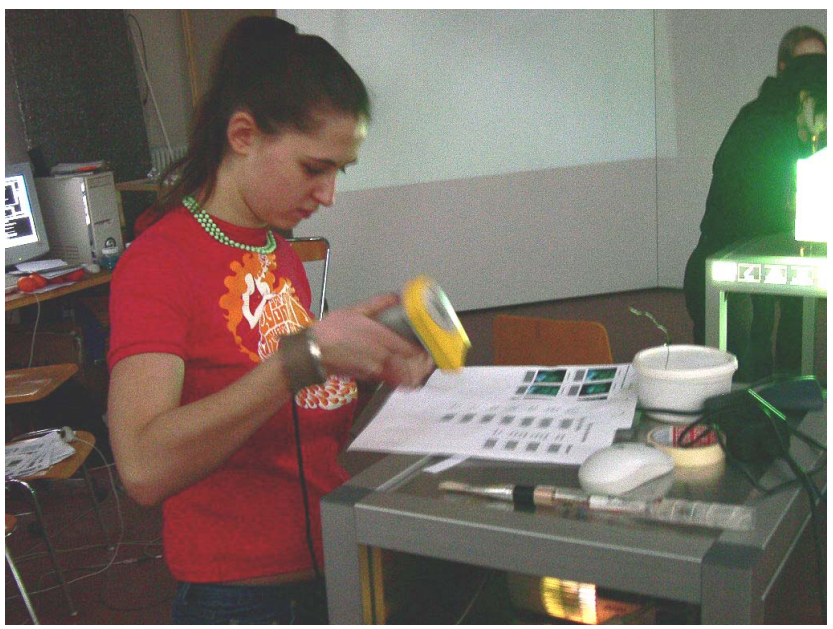

Figure 5. A student working on her projects

Angela is a student at the Academy of Fine Arts working on a project for a new building in Vienna city center. She just came back from an exploration and added a new collection of pictures to the database. She needs to fetch an image she added to the database a few days before. Unfortunately she does not have the Thumbnail Page containing the image. She remembers the color composition: it was red in the middle, green on the bottom and blue on the top side. She picks up some color cubes and places them on the Tangible Image Query surface the way she remembers how the image looked like. The system automatically shows the first nine pictures matching her composition. She does not find the desired one among them. Using the barcode reader and the Query Control Sheet (Figure 4(a)) she browses through the results nine pictures a time, until she finds the desired one. She selects the picture she was looking for (Figure 4(a), image selection highlighted) and sends it to the left side display to see it. Enlarging the picture, she decides to annotate it with some keywords, in order to find it quicker next time. She skims the Ontology Booklet and each time she finds a suitable keyword she toggles it on for the selected picture. She simply scans the correspondent barcode that is printed on the left side of each term (Figure 2, "assign keyword" highlighted). Selected keywords are displayed on the screen for a few seconds in a list below the image. Once she has provided a proper description for the picture she is curious to know which other pictures have a similar description so she scans the barcode that sends the selected picture description to the ontology search (Figure 4(b)). Nine pictures, matching to different degrees the given description, are presented and she again browses the results back and forth. Angela is no longer looking for a particular image, she browses the database just to see available images. By doing so, she finds an interesting picture to be placed aside the original one and she projects it on the right-most screen to see it in more detail. She now has two pictures with similar contents but different color schemes. She sends this second picture back to the Image Query component to find color-scheme-wise similar pictures in the database. The results are surprising, suddenly she has an idea, and finds a connection between, at first sight, different images. She decides to add a keyword to the second image, and does it with just few barcode scans. Having the Ontology Booklet in her hands she starts a new ontology search. Angela can save every interesting image found for the later use. After she has collected enough images, she configures her presentation for tomorrow and prints some of them out just because she likes them - all of it just using barcodes. She saves her configuration on a barcode, prints a reminder page, sticks it into her personal notebook and finally she goes home, certain to have an awesome presentation ready for the next day.

That was achieved by using only the Tangible Image Query table, the Ontology Booklet, the Configuration Poster and the Control Sheet. Those very simple tools, with an intuitive interface, give unlimited possibilities for accessing visual material in a huge database. The setup can be used either to search for a particular image, or just to browse through the database, or to combine both approaches. Furthermore, we experienced a kind of dead-lock when using only one search possibility. When browsing only with keywords, it is impossible to get out of the limited set of images containing requested keywords (or explicitly related keywords). This set can be wide, but it is still limited. The same thing happens when using color-layout search only. If "red spot in the middle" is a search criterion, there will be no results without it. The combination of two search mechanisms gives an elegant way to escape the deadlock. If students are stuck with the red spot image, they can start an ontology search using keywords from one of the "red spot images". The resulting images will not be filtered by the colors but by keywords. Students can continue using keywords, or switch back using a different color scheme or picture.

\section{Related works}

The main achievement we present in this paper is the successful integration of different applications into a complex system that allows seamless transitions between interactions relying on different types of information (i.e., color sketches and keywords) through the use of elementary interfaces. Although individual applications are not innovative, the way we put them all together and supplied a simple and seamless interaction with them is novel. Further information on the conceptual background for our work, and the description of implemented components, may be found 
in [1]. The use of ontology-defined keywords to retrieve images from a database is not novel but provides a way to implicitly to organize ideas. We relate our work to other proposals for querying a database in a tangible way, because that is the way we have really exploited the ontology. Making use of tangible interactions to navigate through information spaces was used for the Navigational Blocks [3], and "physical hypermedia" [6] that was developed for the same domain we are dealing with. Palette [12] is a similar interface to our Thumbnail Pages, and it is also based on barcodes and thumbnails. Another interesting barcode application is for WebStickers [8] where barcodes are used to manipulate Internet bookmarks and to provide them with physical affordances the same way we do with digital media in HMDB. Customizable user interfaces, the general concept that our Configurator and Query Control Sheets seem to fit in, were studied [5] and developed using several tech-

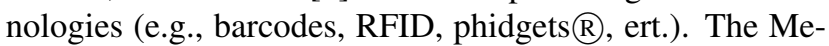
diaBlocks project [14] presents the use of physical markers as handles to digital media and provide a number of appliances. Several systems that fetch images from a database according to image features were also implemented. IBM's QBIC System [13] was one of the first systems. For a complete list of references please refer to the paper on the Tangible Image Query [11].

\section{Conclusion}

We noticed a noticeable impact of our system during experiments. Students used our system not only because they had to (i.e., because the Academy of Fine Arts is a project partner), but because they showed real interest in the possibilities to enhance their work projects. Several facilities and setups were made based on their direct suggestions. Moreover, they invented new ways of interacting with the artifacts, arranging them into choreographic setups.

The needs of students and teachers deeply affected our work. For example, our first version of the ontology tried to be exhaustive, whereas a more flexible one was needed [9]. During project experiments, students were collaborating with us to gain from the experience, in addition to preparing some material for an exam. For this reason we claim the validity of our work based on the qualitative achievements we registered [4]. We noticed improvement in the capability of students to immerse in the environment in a direct and easy way, manipulate artifacts and take care of modeling the environment, and thus the system, around their own needs.

The results from our experiences clearly showed that different strategies to search a large visual database can be applied without forcing the user to stick to a particular context. Users can seamlessly move from color-layout search to keyword-based search without changing the way they interact with the system. Moreover the search results are dis- played all around the user applying the layout that fits best the current work situation. It is important to remark that all the options are available through a paper-based interface that is light-weighted, simple to understand, easy to use and that can dynamically be adapted to new requirements in the environment and kept aligned to system evolution. Users are always able to find what they need in the fastest way, even if they are not the ones who updated the database.

Acknowledgements This work has been supported by the ATELIER project (IST-2001-33064).

\section{References}

[1] T. Binder, G. D. Michelis, M. Gervautz, G. Jacucci, K. Matkovic, T. Psik, and I. Wagner. Supporting configurability in a mixed-media environment for design students. PUC, 8(5):310-325, 2004.

[2] S. Calegari and M. Loregian. Ontologies help finding inspiration: a practical approach in multimedia information management. In PAKM2004, pages 307-318, 2004.

[3] K. Camarata, E. Y.-L. Do, B. R. Johnson, and M. D. Gross. Navigational blocks: navigating information space with tangible media. In IUI '02, pages 31-38, 2002.

[4] P. Ehn et al. ATELIER deliverable D11: Evaluation report of prototyping and scenario experiments. http://atelier.k3.mah.se/publications/ d11_evaluation_report.pdf, 2004.

[5] S. Greenberg and M. Boyle. Customizable physical interfaces for interacting with conventional applications. In UIST 2002, pages 31-40, 2002.

[6] K. Grønbæk, J. F. Kristensen, P. Ørbæk, and M. A. Eriksen. "physical hypermedia": organising collections of mixed physical and digital material. In Hypertext'03, pages 10-19, 2003.

[7] G. Iacucci and I. Wagner. Supporting collaboration ubiquitously: An augmented learning environment for architecture students. In ECSCW' O3, pages 139-158, 2003.

[8] P. Ljungstrand, J. Redström, and L. E. Holmquist. Webstickers: using physical tokens to access, manage and share bookmarks to the web. In DARE 2000, pages 23-31, 2000.

[9] M. Loregian and M. Telaro. Dynamic ontologies and cooperative learning. In Supplements to Proceedings of COOP 2004, pages 73-80, 2004.

[10] K. Matkovic, L. Neumann, J. Siglaer, M. Kompast, and W. Purgathofer. Visual image query. In Smart Graphics 2002, pages 116-123, 2002.

[11] K. Matkovic, T. Psik, I. Wagner, and W. Purgathofer. Tangible image query. In Smart Graphics 2004, pages 31-42, 2004.

[12] L. Nelson, S. Ichimura, E. R. Pedersen, and L. Adams. Palette: a paper interface for giving presentations. In $\mathrm{CHI}$ 99, pages 354-361, 1999.

[13] D. Petkovic et al. Recent applications of IBM's query by image content (QBIC). In SAC 1996, pages 2-6, 1996.

[14] B. Ullmer, H. Ishii, and D. Glas. MediaBlocks: Physical containers, transports, and controls for online media. Computer Graphics, 32:379-386, 1998. 